\title{
High speed spatial encoding enabled by CMOS-controlled micro-LED arrays
}

\author{
(Invited Paper)
Johannes Herrnsdorf, Jonathan J. D. McKendry, Enyuan Xie, Michael J. Strain, Ian M. Watson, Erdan Gu, and Martin D. Dawson
Institute of Photonics, Department of Physics, University of Strathclyde, Glasgow G1 1RD (UK),
Email: johannes.herrnsdorf@strath.ac.uk

\begin{abstract}
Arrays of GaN light-emitting diodes can be used for rapid display of pattern sequences or high speed parallel data transmission using different sites of the array. These operation modes can be combined with each other and are useful for lightfidelity networks with $\mathrm{Gb} / \mathrm{s}$ capacity.
\end{abstract}

\section{INTRODUCTION}

GaN light-emitting diodes (LEDs) are currently being investigated for their properties as transmitters in optical wireless systems. These research efforts are driven by the prospect of combining energy efficient LED lighting with energy efficient high bandwidth local network access. So-called micro-LEDs, i.e. LEDs with a size of $100 \mu \mathrm{m}$ or less, offer a particularly high bandwidth and are therefore an attractive and practical candidate for high speed wireless communications.

Modulation bandwidths in excess of $400 \mathrm{MHz}$ have now been reported by several groups working on micro-LEDs [1], [2], [3], limited by the carrier lifetime. Consequently, data rates up to $5 \mathrm{~Gb} / \mathrm{s}$ have been achieved with a single micro-LED as emitter by employing higher order encoding schemes [4], [5].

However, micro-LEDs do not only offer high bandwidth, but they can readily be fabricated into arrays of individually addressed pixels. These arrays can be interfaced with complementary metal-oxide semiconductor (CMOS) driver electronics [1], [6], [7], [8], thus enabling versatile devices with convenient electronic control.

This presentation will give an overview how state-of-theart LED arrays can be applied to spatially multiplexed data transmission and structured illumination. Such schemes can provide useful services in future light-fidelity environments.

\section{CMOS INTEGRATED LED ARRAYS}

GaN LED arrays can be fabricated with a pixel-to-pixel pitch of $100 \mu \mathrm{m}$ at very high area fill factors up to $98 \%$. The individual elements in such a high density array can be made individually addressable by integration with a CMOS backplane. This is achieved by using substrate-emitting flipchip LEDs that are directly integrated onto the CMOS control chip by bump-bonding as indicated in Fig. 1. Due to the epitaxial structure of the LEDs, the individual contacts in a high fill-factor configuration are the LED anodes and therefore need to be driven by p-MOS transistors.

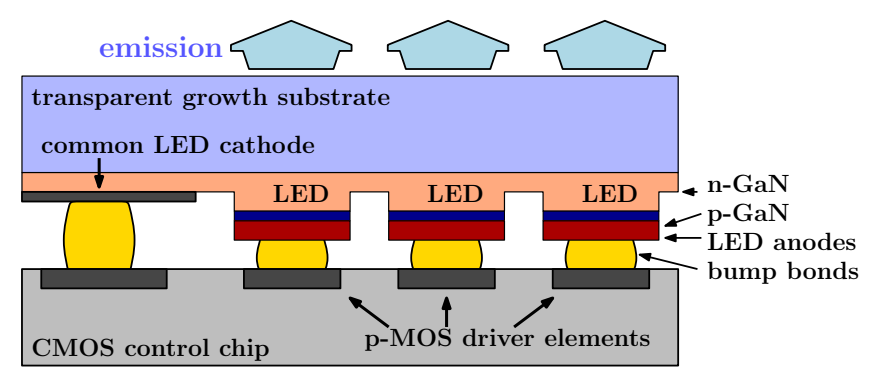

Fig. 1. Schematic of a flip-chip LED array integrated with a p-MOS chip through bump bonding.

P-MOS driven LED arrays have been fabricated in dimensions of $16 \times 16$ and $10 \times 40$ elements and deliver optical output powers up to $4 \mathrm{~mW} /$ pixel [9].

If a high fill-factor and/or large number of LEDs is not required, then the LED array can be fabricated with individual cathode contacts, enabling driving with n-MOS transistors. In this case, the LEDs are contacted to the CMOS chip via wire bonds. We have fabricated n-MOS controlled LED arrays with a dimension of $6 \times 6$ [10].

\section{DATA TRANSMISSION IN SISO AND MIMO CONFIGURATION}

Each LED in the array can carry an individual data signal. Thereby, several data streams are spatially distributed across the chip. There are different ways how this spatially encoded information is transmitted through space and then received. In the simplest case, an imaging system is used that images each individual LED onto one single detector. Such a single-input single-ouput (SISO) configuration has been implemented with p-MOS controlled $16 \times 16$ arrays. Up to 16 data streams were transmitted in parallel at an aggregate data rate of $1.2 \mathrm{~Gb} / \mathrm{s}$. This demonstration used a very practical implementation where the entire device was powered and operated through a USB cable. The physical limitations of the device were tested at voltages higher than the $5 \mathrm{~V}$ available from USB and with high speed bit error rate test equipment. In this case, an aggregate data rate of $1.5 \mathrm{~Gb} / \mathrm{s}$ was transmitted in 4 parallel channels using on-off-keying [11].

On the other hand, in a multiple-input multiple-output (MIMO) system the optical emission of the different LEDs 


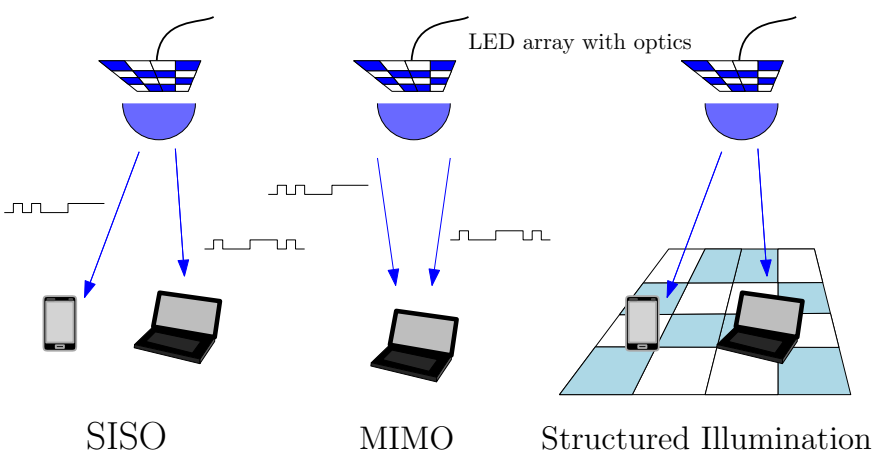

Fig. 2. Schematic illustration of the different operation modes: single-input single-output (SISO), multiple-input multiple-output (MIMO) and structured illumination through a rapidly displayed illumination pattern sequence.

overlaps at the receiver position as indicated in Fig. 2. A multi-element detector on the receiving device is then used to retrieve the individual data streams. $2 \times 2$ and $3 \times 3 \mathrm{MIMO}$ systems were implemented with n-MOS controlled LED arrays and the maximum data rates were $1.8 \mathrm{~Gb} / \mathrm{s}$ and $6.95 \mathrm{~Gb} / \mathrm{s}$, respectively [12].

\section{Positioning SYSTEM BASED ON STRUCTURED ILLUMINATION}

Individually controlled high-density arrays can not only carry individual data signals on individual LEDs, but they can also be used to project a rapidly displayed time-sequence of illumination patterns onto the illuminated scene. E.g. a $10 \times 40$ LED array can display such a sequence at a frame rate of $30 \mathrm{kfps}$, limited by the electronic driver implementation and well beyond visual flicker recognition. Any device with a photodetector within the illuminated scene as indicated in Fig. 2 can monitor the signal it receives for each pattern. By appropriate choice of the pattern sequence, the devices can determine their position from the time-sequence optical signal they receive [13].

This positioning method is very undemanding on the receiver end. A single photodiode can be used to receive the signal and the computationally cheap decoding algorithm can be implemented without using any multiplications or divisions. The number of patterns required for positioning scales logarithmically with the number of emitting LEDs and therefore positioning within on the order of one millisecond is feasible.

This type of positioning system has been combined with SISO data transmission. In this case, the LED array switched between two operation modes. First, the pattern sequence for positioning was diplayed until the detectors placed in the scene had determined their position. Then, the array switched to a beaming mode, where only the LEDs corresponding to the individual detector positions were activated. Each of these LEDs carried an individual data signal, thus providing the different SISO channels.

\section{CONCLUSION}

CMOS-interfaced micro-LED arrays can be operated in several operation modes that are useful for light-fidelity networks. In SISO and MIMO mode, data can be transferred to one or several devices at $\mathrm{Gb} / \mathrm{s}$ data rates. Furthermore, these arrays can provide an intelligent environment based on structured illumination accessible to devices with a photodetector. Device positions can be determined by a simple method and individual high data rate optical wireless connections can be made to mobile devices.

\section{ACKNOWLEDGMENT}

The authors thank the Engineering and Physical Sciences Research Council for funding under the grants EP/K00042X/1, UPVLC, http://up-vlc.photonics.ac.uk/ and EP/M01326X/1, QuantIC. We acknowledge support from our project partners, including in particular the groups of Robert Henderson and Harald Haas at the University of Edinburgh and Dominic O'Brien at the University of Oxford.

\section{REFERENCES}

[1] J. J. D. McKendry et al., "Visible-Light Communications Using a CMOS-Controlled Micro-Light-Emitting-Diode Array," J. Lightwave Technol., vol. 30, no. 1, pp. 61-67, 2012.

[2] C.-L. Liao et al., "High-Speed Light-Emitting Diodes Emitting at $500 \mathrm{~nm}$ With 463-MHz Modulation Bandwidth," IEEE Electron. Dev. Lett., vol. 35, no. 5, 2014.

[3] P. P. Maaskant et al., "High-Speed Substrate-Emitting Micro-LightEmitting Diodes for Applications Requiring High Radiance," Appl. Phys. Express, vol. 6, p. 022102, 2013.

[4] D. Tsonev et al., "A 3-Gb/s Single-LED OFDM-Based Wireless VLC Link Using a Gallium Nitride $\mu$ LED," IEEE Photon. Technol. Lett., vol. 26 , no. 7 , pp. 637-640, 2014

[5] R. X. G. Ferreira et al., "High bandwidth GaN-based micro-LEDs for multi-Gbps visible light communications," submitted to IEEE Photon. Technol. Lett.

[6] J. J. D. McKendry et al., "Individually Addressable AlInGaN MicroLED Arrays With CMOS Control and Subnanosecond Output Pulses," IEEE Photon. Technol. Lett., vol. 21, no. 12, pp. 811-813, 2009.

[7] Z. J. Liu et al., "Monolithic LED Microdisplay on Active Matrix Substrate Using Flip-Chip Technology," J. Sel. Topics Quantum Electron., vol. 15, no. 4, pp. 1298-1302, 2009.

[8] J. Day et al., "III-Nitride full-scale high-resolution microdisplays," Appl. Phys. Lett., vol. 99, p. 031116, 2011.

[9] J. Herrnsdorf et al., "Active-Matrix GaN Micro Light-Emitting Diode Display with Unprecedented Brightness," IEEE Trans. Electron Dev., vol. 62, no. 6, pp. 1918-1925, 2015.

[10] S. Rajbhandari et al., "High-Speed Integrated Visible Light Communication System: Device Constraints and Design Considerations," IEEE J. Sel. Areas Comm., vol. 33, no. 9, pp. 1750-1757, 2015.

[11] S. Zhang et al., "1.5 Gbit/s Multi-Channel Visible Light Communications Using CMOS-Controlled GaN-Based LEDs," J. Lightwave Technol., vol. 31, no. 9, pp. 1211-1216, 2013.

[12] S. Rajbhandari et al., "A 7 Gbps Integrated Multiple Input Multiple Output Visible Light Communication Demonstrator," submitted to J. Lightwave Technol.

[13] J. Herrnsdorf et al., "Concept of a GaN-LED-based positioning system using structured illumination," in IEEE Photonics Conference, 2015. 